\title{
A protocol for the process evaluation of a multi-centre randomised trial to compare the effectiveness of geriatrician-led admission avoidance hospital at home versus inpatient admission
}

\author{
Petra Mäkelä ${ }^{1,4^{*}}$ (D, Mary Godfrey ${ }^{2}$, Andrea Cradduck-Bamford ${ }^{1}$, Graham Ellis ${ }^{3}$ and Sasha Shepperd ${ }^{1,4^{*}}$
}

\begin{abstract}
Background: Attempts to design services to support the delivery of healthcare closer to home have taken various forms as countries respond to an increase in hospital admission rates for older people, who are at risk of hospitalacquired morbidity, prolonged lengths of stay and readmission. Evidence to support the development of these services is limited. We are conducting a process evaluation, alongside a UK multi-site randomised trial, to understand the contexts and practices of implementing geriatrician-led admission avoidance hospital at home services and to explore ways that the intervention might be effective, under what conditions, for whom, and how it differs from inpatient care.
\end{abstract}

Methods: We are interviewing patients and their caregivers, from sites that are purposively sampled from participating National Health Service (NHS) trusts across the UK. We are also visiting sites to observe local processes and discuss the establishment and running of services with a range of multidisciplinary staff, managers, commissioners, primary care and social services representatives. We aim to interview approximately 36 patients and their caregivers with experience of hospital at home or inpatient services; 12 at each of three sites. We will use a content analysis approach to explore data across participants, services and sites.

Discussion: This process evaluation will enable evaluation of implementation processes prior to knowing trial outcomes. We encompass domains of reach, delivery, change, context and response to the intervention by patients, their carers, health professionals and the health system.

Trial registration: ISRCTN60477865. Registered on 10 March 2014. Trial sponsor: University of Oxford. Version 3.1, registered on 14 June 2016.

Keywords: Process evaluation, Comprehensive Geriatric Assessment, Hospital at home, Admission avoidance

* Correspondence: p.makela@nhs.net; sasha.shepperd@ndph.ox.ac.uk

${ }^{1}$ Nuffield Department of Population Health, Richard Doll Building, University

of Oxford, Old Road Campus, Oxford OX3 7LF, UK

Full list of author information is available at the end of the article

(c) The Author(s). 2018 Open Access This article is distributed under the terms of the Creative Commons Attribution 4.0 International License (http://creativecommons.org/licenses/by/4.0/), which permits unrestricted use, distribution, and reproduction in any medium, provided you give appropriate credit to the original author(s) and the source, provide a link to the Creative Commons license, and indicate if changes were made. The Creative Commons Public Domain Dedication waiver (http://creativecommons.org/publicdomain/zero/1.0/) applies to the data made available in this article, unless otherwise stated. 


\section{Background}

The demographic change of population ageing has increased the demand for healthcare services internationally $[1,2]$. Older people who present to healthcare services with an acute event may be admitted to hospital in the absence of alternative responses [3]. Older people often have complex needs and, when experiencing a health event, are vulnerable to adverse outcomes, a state commonly referred to as frailty [4-6]. As an inpatient, an older person with frailty may contend with hospital-acquired morbidity, a prolonged length of stay and a likelihood of readmission to hospital [4, 7-9].

In the UK, an emphasis on avoiding admission to hospital has gained momentum alongside an annual rise in emergency admissions [10, 11]. Growing demands for costly inpatient care and an increasingly constrained budget for the National Health Service (NHS) [12] have led to a policy focus on moving care away from hospitals to the community $[3,11,13,14]$. Geriatrician-led admission avoidance hospital at home $(\mathrm{HAH})$ is a model that is emerging internationally, referring to hospital-level treatment that is delivered by healthcare professionals (sometimes as a hospital outreach service) to patients who remain in their own homes $[15,16]$. This is guided by the principles of Comprehensive Geriatric Assessment (CGA), a cornerstone of care for older people requiring hospital-level healthcare [17-19]. CGA is defined as 'a multidimensional, multidisciplinary process which identifies medical, social and functional needs, and the development of an integrated/coordinated care plan to meet those needs' [20].

We describe the methods of a process evaluation that we are conducting alongside a multi-site randomised trial of geriatrician-led admission avoidance HAH compared with hospital admission [21]. The main outcome will be 'living at home' at 6 months' follow-up and other outcomes will include the incidence of delirium, mortality, new long-term residential care, cognitive impairment, activities of daily living, quality of life, quality-adjusted survival, length of stay, readmission or transfer to hospital and resource use. The randomised controlled trial (RCT) protocol has been described in detail previously [21].

\section{Aims and objectives}

Within the context of a multi-site randomised trial we aim to expand understandings of what works in geriatrician-led $\mathrm{HAH}$ and inpatient settings, for whom, in what respects, to what extent, in what contexts, and how. The objectives are:

- To explore components, practices and experiences of CGA in HAH and inpatient settings

- To describe key elements of trial contexts and explore how these might affect implementation of the intervention and of the RCT
- To identify unanticipated consequences and aspects of the trial that are not necessarily captured quantitatively

\section{Methods}

This a priori process evaluation is informed by the UK's Medical Research Council (MRC) guidance [22, 23] and a framework for evaluation within trials [24]. Ethical approval for the trial and process evaluation was given by the Research Ethics Committee England, Wales and Northern Ireland (14/WA/1081) and Scotland (14/SS/1046).

\section{Setting}

We have selected three sites from those participating in a multi-site trial (two NHS trusts in England and one in Scotland, see Fig. 1) enabling comparison of implementation and a range of perspectives. These sites represent differing geographical areas, service compositions, populations and organisational arrangements that might influence the delivery and effectiveness of the intervention [23].

\section{Design}

The process evaluation design follows three interlinked stages: (1) determining existing theory and objectives underlying the complex intervention, (2) refining the research questions and (3) generating data.

\section{Existing theory and objectives underlying the complex intervention}

A logic model of CGA (Fig. 2) outlines key aspects that may be shared across different settings and displays the relationship between resources, activities and intended results [25]. The accompanying programme theory proposes that implementation of CGA through geriatrician-led admission avoidance $\mathrm{HAH}$, instead of an acute hospital admission, will lead to a greater improvement in health outcomes due to the hospital environment potentially limiting the processes of recovery.

\section{Process evaluation questions}

Our research questions address implementation of CGA in HAH and inpatient settings, and implementation of the RCT across trial contexts. We are guided by the Consolidated Framework for Implementation Research (CFIR) [26, 27], which specifies five key domains: intervention characteristics, outer setting (factors external to the organisation), inner setting (characteristics of the organisation), characteristics of the individuals involved in the intervention, and the processes of implementation [26] (see Table 1). Our intention is to undertake the process evaluation with sufficient flexibility to allow us to identify and address additional questions that may arise during the course of the study, recognising that local and broader aspects of 


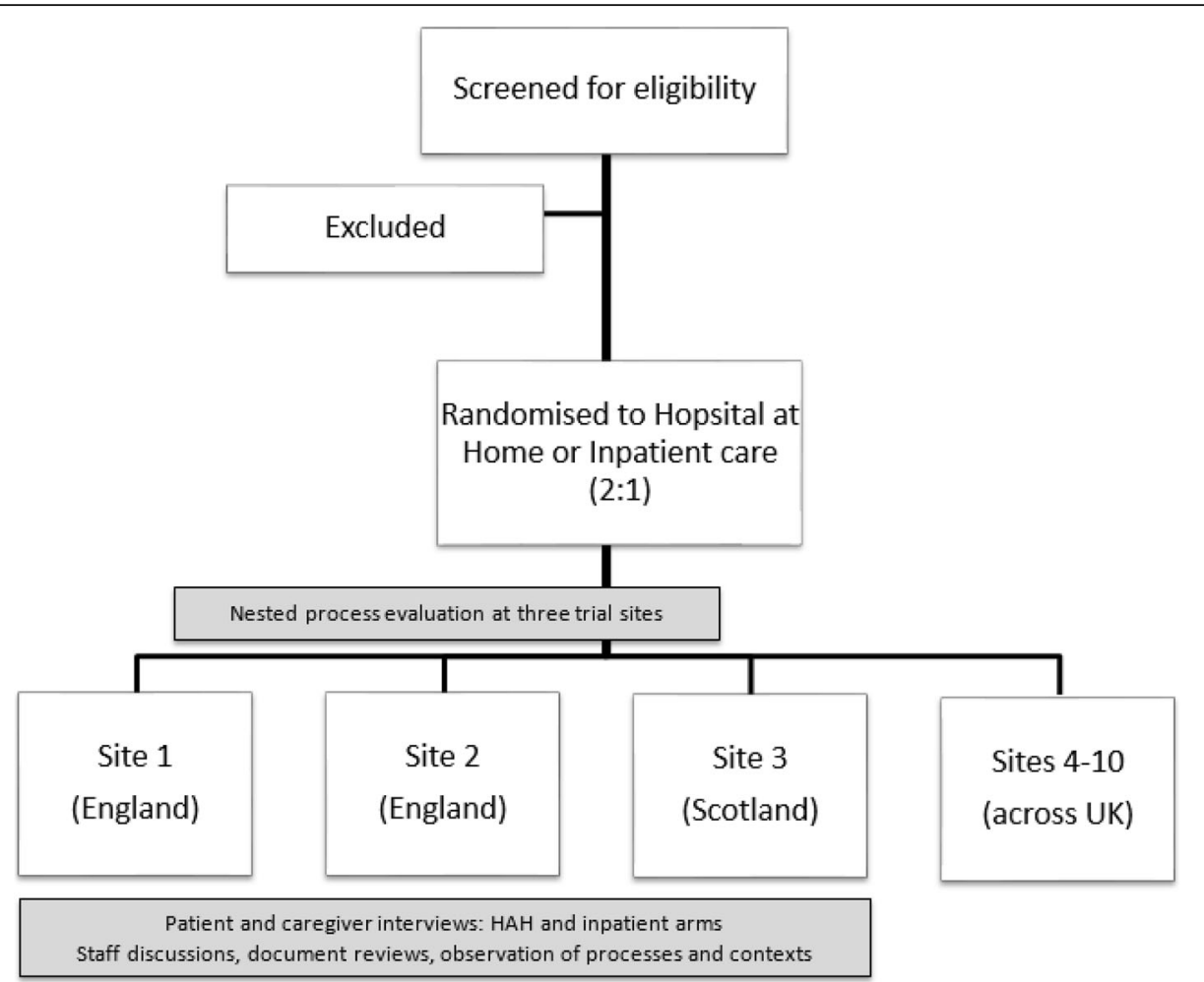

Fig. 1 Process evaluation overview

these domains are likely to become more salient as the work iteratively develops $[28,29]$.

\section{Generating data}

We generate qualitative data to explore the research questions through field visits and by undertaking discussions with a range of multidisciplinary staff, managers, commissioners, representatives of primary care and social services. In addition, we conduct semi-structured interviews with a purposive sample of patients who have been randomised to the trial and their caregivers. Data generation is conducted iteratively over time to develop relationships with each site.

Field visits Field visits are being conducted to find out about how the services are organised, the reach of the intervention, and interfaces with related services delivering health and social care. We review policy and guidance documents referred to by staff, service assessment documents, discharge templates, protocols or other documentation considered important to the services. We undertake in-depth discussions with a range of staff

\begin{tabular}{|c|c|c|c|c|c|c|}
\hline Problem & Evidence & Inputs & Activities & Outputs & Outcomes & Impact \\
\hline $\begin{array}{l}\text { - Growing } \\
\text { demand for } \\
\text { acute inpatient } \\
\text { care } \\
\text { - Risk of } \\
\text { functional } \\
\text { decline and } \\
\text { other } \\
\text { complications } \\
\text { for older } \\
\text { people in } \\
\text { hospital }\end{array}$ & $\begin{array}{l}\text {-Admission } \\
\text { avoidance } \\
\text { HAH may be } \\
\text { effective } \\
\text { alternative to } \\
\text { acute inpatient } \\
\text { care } \\
\text { - Limited } \\
\text { evidence to } \\
\text { determine } \\
\text { which older } \\
\text { people may } \\
\text { benefit }\end{array}$ & $\begin{array}{l}\text {-Geriatrician- } \\
\text { led multi- } \\
\text { disciplinary } \\
\text { team } \\
\text { - Resources and } \\
\text { team skills for } \\
\text { hospital level } \\
\text { care in } \\
\text { patients' } \\
\text { homes } \\
\text {-Accessible } \\
\text { diagnostic and } \\
\text { community } \\
\text { services } \\
\text { including } \\
\text { social care }\end{array}$ & $\begin{array}{l}\text { - Coordinated } \\
\text { teamwork } \\
\text { through MDT } \\
\text { meetings } \\
\text { - CGA model } \\
\text { underpinning } \\
\text { multi- } \\
\text { dimensional } \\
\text { assessment } \\
\text { and } \\
\text { management } \\
\text { - Involvement of } \\
\text { patients and } \\
\text { caregivers }\end{array}$ & $\begin{array}{l}\text { - Optimised } \\
\text { medical, social, } \\
\text { psychological } \\
\text { and functional } \\
\text { needs of } \\
\text { patients } \\
\text { - Family and } \\
\text { caregivers } \\
\text { supported and } \\
\text { informed }\end{array}$ & $\begin{array}{l}\text { - Reduction in } \\
\text { acute hospital } \\
\text { admissions } \\
\text { and inpatient } \\
\text { occupancy } \\
\text { - Reduction in } \\
\text { need for } \\
\text { longterm } \\
\text { residential } \\
\text { care }\end{array}$ & $\begin{array}{l}\text { - Improved } \\
\text { health and } \\
\text { wellbeing for } \\
\text { older people } \\
\text { with frailty } \\
\text { - Increased } \\
\text { likelihood of } \\
\text { older people } \\
\text { remaining in } \\
\text { own homes }\end{array}$ \\
\hline
\end{tabular}

Fig. 2 Simplified logic model for Comprehensive Geriatric Assessment (CGA) 
Table 1 Research questions

\begin{tabular}{ll}
\hline Domain & Focus \\
\hline Context & Outer setting: what is the local, regional and national \\
context? How may social, political and economic & contexts influence implementation? \\
- Inner setting: how do organisational and service \\
structures, cultures and relationships influence \\
implementation? \\
- What are the characteristics of those delivering and \\
Implementation \\
settings? \\
- What are the intervention characteristics and \\
processes of implementation in each setting, and \\
how do these relate to the experiences and \\
engagement of patients and caregivers? \\
- How do these aspects of implementation relate \\
to the existing programme theory and logic model \\
for CGA (Fig. 2)? \\
- How are individuals recruited and by whom? \\
- Are those recruited to the RCT representative of the \\
overall 'real world' target population? \\
- How do RCT implementation processes differ across \\
settings? \\
- How are RCT processes sustained or threatened \\
over time? \\
- Are there unintended consequences in processes \\
and outcomes related to involvement in RCT, to \\
the intervention or to other aspects of care?
\end{tabular}

CGA Comprehensive Geriatric Assessment, HAH hospital at home, $R C T$ randomised controlled trial

in both settings at each of the three sites, and capture field notes with the use of an observation framework attending to environment, ways of working, roles, interactions, relationships, activities, documents, methods of communication and other aspects that facilitate teamwork, clinical processes and patient or caregiver interactions.

Healthcare professionals We purposively sample healthcare professionals to achieve diversity of roles and experience, aiming to yield 'information-rich' discussions [30]. In addition, we use snowball sampling where managers or clinical leads identify clinicians and support staff who are known to have suitable experience of the service, for whom brief 'release' from workload activities has been agreed for their participation in a discussion with the qualitative researcher [31]. Discussions are undertaken individually or with small groups, according to staff time constraints and availabilities. We aim to continue staff discussions until data generated enable interpretations and credible explanations that address the research questions [32].

Multidisciplinary staff invited to discussion at each of the three sites include managers and clinical leads for services participating in the RCT, allied health professionals across a range of seniorities, staff nurses, ward sisters and matrons, healthcare assistants, rehabilitation support workers, consultant geriatricians, junior doctors, physician assistants, primary care representatives, pharmacists, social workers, administrative and domestic services staff, and healthcare commissioners in England and Health and Social Care Partnerships in Scotland. We also discuss RCT implementation with principle investigators and research coordinators at each site. We capture aspects of variation between staffing complements; for example, whether teams include pharmacists or social workers. In addition, we anticipate that differences in team complements will be dynamic through the study and we explore contextual factors driving changes, vacancies or restructuring within services. Collection of contextual data will enable exploration of differences including associated service provision, perceptions of the intervention and drivers of change perceived in the healthcare environment.

Patients and caregivers We invite a sample of patients randomised to $\mathrm{HAH}$ or inpatient care from each of the three sites to participate in qualitative interviews. We include people with and without family caregivers or formal carers and, where available, we invite family caregivers to take part in the interview also. We approach patients with cognitive or communication impairment, including people who may lack or have variable capacity to consent to the interview study, if they have appropriate support available to take part. Capacity assessment follows the principles of Mental Capacity Act (2005) in England [33] and the Adults with Incapacity Act (2000) in Scotland [34].

In view of the practicalities of arranging interviews in patients' homes across geographically dispersed sites, and identifying patients who have been randomised to each arm of the RCT (with a 2:1 randomisation ratio for intervention to control), convenience sampling is also necessary [31]. The planned sample size is six patients (with a family member, caregiver or significant other), for each arm of the RCT, at each of the three sites (i.e. approximately 36 interviews in total). We are flexible to the potential need for a small number of further interviews to pursue promising lines of enquiry not anticipated in advance. We review the recruitment strategy iteratively, to ensure adequate sampling and to address practical issues that may affect timely access to participants.

The qualitative researcher approaches patients regarding the interview study following initial contact made by the trial research nurse or coordinator at the site, who confirms that patients are medically stable, are not receiving end of life care and that there are no other identified reasons why approach may be inappropriate or intrusive for the patient and family. The research nurse or coordinator provides written information about the interview study and confirms participants' agreement for further contact. The qualitative researcher then discusses the interview study with the patient or consultee, 
answers questions and arranges a convenient time for the interview. The researcher arranges interviews around the time of discharge, or soon after discharge, from the healthcare service participating in the RCT, and carries these out in patients' homes or on inpatient wards. We emphasise that patients and caregivers are not under any obligation to participate in the interview study and that, if they agree to take part, they are free to withdraw at any time without having to provide an explanation and without any effect on their usual care. We explain that all personal information will be anonymised and that researchers are not connected to patients' clinical care.

\section{Interview processes}

The researcher records patient and caregiver interviews using an encrypted digital audio-recording device, subject to permission by participants. Interviews last between 30 and $60 \mathrm{~min}$. We use topic guides as prompts for the semi-structured interviews, with versions adapted for experiences of $\mathrm{HAH}$ or inpatient care. We developed the topic guides from earlier iterations informed by focus group discussions with older people and family caregivers who had experience of $\mathrm{HAH}$ or admission to hospital. Within interviews, the researcher avoids the use of 'jargon' terms that may be unfamiliar to participants (such as 'CGA' [35]), but instead uses phrases that may illustrate aspects of experience; for example, 'Can you describe ways the healthcare team supported you?'. Areas of exploration within interviews include:

- Participants' accounts of their presenting event and means of accessing acute healthcare

- Perceptions of interactions with healthcare professionals and other staff throughout the trajectory of service input for their presenting episode, from assessment to discharge and any follow-up received

- Whether any documentation was provided to patients and caregivers and, if so, how they perceived and used this (e.g. service information leaflets, goal sheets, medication information, discharge letters)

- How patients understood the intervention or other measures to have contributed to recovery from their presenting event and their ability to continue to manage after discharge

- Caregivers' perceptions of positive and negative aspects of the healthcare experience and how effectively they perceived the patient's and their own needs to have been addressed

- How and where they received input from healthcare services, and how healthcare professionals communicated or discussed transitions with them
We use a flexible approach and consider individuals' specific healthcare experiences as conceived by participants themselves; for example, experiences that have extended to transitions between different parts of the health and social care system. The interview is adapted if topics appear to be causing undue distress. Where appropriate, the interview ceases and the researcher offers a subsequent interview date; for example, if events external to the interview have contributed to distress and if the participant would like to complete the discussion at a later stage. The researcher will follow up instances of distress with the site research team and principle investigator to determine any actions required.

\section{Ethical issues}

Patients who have been recruited to the RCT may have reduced, fluctuating or absent capacity to consent to trial participation and requirements [21]. As capacity to consent refers to a time- and decision-specific ability [36], capacity is assessed for inclusion in the interview study as an additional consideration to consent for overall RCT participation. If assessment indicates that a patient lacks capacity to consent to participate, the researcher discusses the interview study with a personal consultee who is 'engaged in caring for the person or is interested in their welfare' [33] and is involved in making decisions that reflect the patient's views and values [37]. The researcher also invites the personal consultee to the interview study, following their informed consent, to share their own experiences and perspectives and to support the patient.

Personal consultees may support people with cognitive or communication impairment through the generation of 'scaffolds' in conversation, which can be used by the person in making sense of the topic under discussion, assisting them to find words or recognise reference points [38], p.341. We pay attention to use of terminology within interviews, particularly ways participants choose to refer to themselves and their respective roles, as questions that emphasise caregiving within families may differ from individuals' usual ways of thinking about their mutual relationships [39]. As the majority of interviews take place in patients' homes (or professionals' places of work), the researcher becomes a 'guest' in these spaces and allows participants to set their own pace in discussions [40].

\section{Reflexivity}

The qualitative researcher captures observations and impressions in field notes soon after interactions and field visits, including contextual information and reflections on potential influences of the researcher's own perspectives. [40, 41]. 


\section{Data management and analysis}

Audio data is fully transcribed by a professional agency and is checked by the researcher for accuracy. We allocate pseudonyms to all participants and anonymise other potential identifiers within transcripts. All personally identifiable data is stored in password-protected files in a secure environment. Field notes pertaining to site visits and observations from interviews in home settings are managed anonymously and analysed. We use a spreadsheet to log all 'raw' data generated, to detail progress and highlight potential gaps in the evaluation. Data are subsequently managed in NVivo 11 (QSR international) to aid sorting and organising of the large volume and different types of data, and to facilitate analysis.

\section{Analysis of contextual data}

We will produce a narrative, descriptive account of the organisation of services at each site studied, drawing on data collated in field notes from observations during visits, review of formal documents relating to organisation and delivery, and notes from discussions with a range of healthcare professionals. We focus on factors guided by CIFR domains: intervention characteristics, outer setting (external to the organisation), inner setting (within the organisation), individuals involved and the processes of implementation. Changes to staffing and service organisation are captured, which might have impact on the delivery of healthcare. The evaluation of trial implementation explores sustainability of activities and strategies used at the interface between service delivery (in varied clinical settings) and RCT protocol requirements.

\section{Analysis of qualitative interviews with patients and caregivers}

We plan to undertake content analysis of transcribed interviews to interpret data through identifying, organising, describing and reporting themes or patterns [42, 43]. The analysis involves an iterative and reflective process that develops over time, moving back and forward between data generation and analysis, allowing issues identified to inform subsequent data generation and deepen areas of exploration.

We use a framework approach to facilitate the content analysis, address the research questions, and enable comparison within and between participants' control and intervention experiences [44, 45]. We will develop an initial coding framework deductively from literature on CGA and $\mathrm{HAH}$, the existing logic model and programme theory. We will expand this inductively from analysis of field notes and interviews and will develop the framework through iterative analysis across data sources, with ongoing discussion among the research group, aiming to capture new and unexpected issues that arise [44]. The framework approach to organising content analysis provides a clear trail of evidence for the credibility of the study [46], allowing management of large volumes of data while retaining connection to original sources, to avoid 'decontextualisation and fragmentation' of participants' accounts [26], p.810. Extracts of raw data will be embedded within the interpretive narrative, to illustrate complexities that move beyond a description of data $[43,46]$.

\section{Dissemination}

We will disseminate findings in a final report, peerreviewed publications in academic and practitioner journals, and through conference presentations. We will engage with healthcare professionals' and patient and caregivers' groups to facilitate translation of findings, to inform clinical practice and to assist in contextualisation of trial findings.

\section{Discussion}

This protocol outlines the design, data generation and analysis plan for a process evaluation embedded in a multi-centre trial of geriatrician-led admission avoidance HAH compared with hospital admission. The use of qualitative research facilitates understanding of how the intervention works in practice and aims to identify aspects perceived important by patients, caregivers and practitioners. Limitations of the process evaluation include prospective decisions about the focus within sites, though the design incorporates flexibility to respond to and expand research questions as the process evaluation progresses.

\section{Abbreviations \\ CFIR: Consolidated Framework for Implementation Research; \\ CGA: Comprehensive Geriatric Assessment; HAH: Hospital at home; MDT: Multidisciplinary team; MRC: Medical Research Council; NHS: National Health Service; RCT: Randomised controlled trial}

\section{Acknowledgements}

The overall supervision of the trial and process evaluation is carried out by the Trial Steering Committee. The sponsor is the University of Oxford. We are grateful to Bradford Teaching Hospitals NHS Foundation Trust, Guy's and St Thomas' NHS Foundation Trust, University Hospital Monklands NHS Lanarkshire, and John Young, Maj Pushpangadan and Rebekah Schiff for supporting the conduct of the process evaluation.

\section{Funding}

This research was funded by the NIHR Health Services and Delivery Research Programme (project number 12/209/66). Department of Health disclaimer: the views expressed are those of the author(s) and not necessarily those of the NHS, the NIHR or the Department of Health.

\section{Authors' contributions}

$\mathrm{PM}$ is a qualitative researcher who is undertaking the data collection and led writing of the process evaluation protocol manuscript. MG is a grant holder, is leading the process evaluation and contributed to the manuscript. ACB is the trial manager, has contributed to the implementation of the trial and to the protocol manuscript. GE is a grant holder and principle investigator at one of the sites. SS is the chief investigator, is the grant holder, identified the gap in the evidence and is leading the implementation of the trial and process evaluation and contributed to the manuscript. All authors read and approved the final manuscript. 


\section{Ethics approval and consent to participate}

The design of this process evaluation is included within the ethics application and overall protocol for the RCT [21]. Ethical approval for the trial was given by the Research Ethics Committee England, Wales and Northern Ireland (14/WA/1081) and Scotland (14/SS/1046). Participants who meet the inclusion criteria and verbally agree to participate are asked to sign a consent form. If a participant has difficulty completing the consent form due to visual impairment or frailty, but is capable of informed consent, a witness signature is sought from a next of kin or medical professional not working within the trial team to confirm the consent process was appropriately performed. We are recruiting adult participants with cognitive impairment/ dementia who are unable to consent for themselves. We consider that this is necessary because, if recruitment is restricted to patients with capacity, such a restriction would lead to an unrepresentative study sample. The consent process takes into account the implications of the Mental Capacity Act (2005) in England, Wales and Northern Ireland and the Adults with Incapacity Act (2000) in Scotland. A relative, friend or Independent Mental Capacity Advocate is involved in making a decision in the best interests of individuals if they do not have capacity to give consent. If necessary, this consultee consent is taken verbally over the phone and the paperwork sent to the consultee to be signed to record consent. We reassess capacity at follow-up visits and re-consent a participant if their capacity changes between baseline and follow-up. Participants, or their representatives, are asked to sign and date the latest approved version of the Informed Consent Form before any trial-specific procedures are performed. The trial manager ensures that all sites are aware of any amendments.

\section{Consent for publication}

Not applicable.

\section{Competing interests}

The authors declare that they have no competing interests.

\section{Publisher's Note}

Springer Nature remains neutral with regard to jurisdictional claims in published maps and institutional affiliations.

\section{Author details}

${ }^{1}$ Nuffield Department of Population Health, Richard Doll Building, University of Oxford, Old Road Campus, Oxford OX3 7LF, UK. ${ }^{2}$ Institute of Health Sciences, University of Leeds, Leeds LS2 9LJ, UK. ${ }^{3}$ Monklands Hospital, Monkscourt Avenue, Airdrie ML6 0JS, UK. ${ }^{4}$ London School of Hygiene and Tropical Medicine, Keppel Street, London, UK.

\section{Received: 6 July 2018 Accepted: 21 September 2018}

Published online: 19 October 2018

\section{References}

1. WHO. World Report on Ageing and Health. Geneva: World Health Organization; 2015.

2. Rizzuto D, Melis RJF, Angleman S, Qiu C, Marengoni A. Effect of chronic diseases and multimorbidity on survival and functioning in elderly adults. J Am Geriatr Soc. 2017;65:1056-60.

3. King's Fund. Ten priorities for commissioners. The King's Fund 2015. https:// www.kingsfund.org.uk/publications/articles/transforming-our-health-caresystem-ten-priorities-commissioners/summary. Accessed 10 Jan 2018.

4. Inouye SK, Studenski S, Tinetti ME, Kuchel GA. Geriatric syndromes: clinical, research, and policy implications of a core geriatric concept. J Am Geriatr Soc. 2007:55:780-91.

5. Clegg A, Young J, Iliffe S, Rikkert MO, Rockwood K. Frailty in elderly people. Lancet. 2013;381:752-62.

6. Lally F, Crome P. Understanding frailty. Postgrad Med J. 2007;83:16-20

7. Parker SG, McLeod A, McCue P, Phelps K, Bardsley M, Roberts HC, et al. New horizons in Comprehensive Geriatric Assessment. Age Ageing. 2017;46:713-21. https://doi.org/10.1093/ageing/afx104.

8. Covinsky KE, Palmer RM, Fortinsky RH, Counsell SR, Stewart AL, Kresevic D, et al. Loss of independence in activities of daily living in older adults hospitalized with medical illnesses: increased vulnerability with age. J Am Geriatr Soc. 2003;51:451-8.

9. Liu B, Moore JE, Almaawiy U, Chan W-H, Khan S, Ewusie J, et al. Outcomes of Mobilisation of Vulnerable Elders in Ontario (MOVE ON): a multisite interrupted time series evaluation of an implementation intervention to increase patient mobilisation. Age Ageing. 2018;47:112-9.

10. Morris J. Combined Performance Summary: QualityWatch. 2018. http://www.qualitywatch.org.uk/blog/combined-performance-summarynovemberdecember-2017\#. Accessed 11 Jan 2018.

11. The Nuffield Trust. Shifting the balance of care. 2017. https://www. nuffieldtrust.org.uk/files/2017-02/shifting-the-balance-of-care-report-webfinal.pdf. Accessed 11 Jan 2018.

12. Appleby J. 10 crucial trends: Quality in the NHS 2009 to 2017 | QualityWatch. QualityWatch 2017. http://www.qualitywatch.org.uk/blog/10crucial-trends-quality-nhs-2009-2017. Accessed 12 Jan 2018.

13. NHS England. NHS Five Year Forward View. 2014. https://www.england.nhs. uk/five-year-forward-view/. Accessed 2 Jan 2018.

14. Monitor. Moving healthcare closer to home: clinical review. 2015. https:// www.gov.uk/government/uploads/system/uploads/attachment_data/file/ 459268/Moving_healthcare_closer_to_home_clinical_review.pdf. Accessed 10 Jan 2018.

15. Shepperd S, lliffe S, Doll HA, Clarke MJ, Kalra L, Wilson AD, et al. Admission avoidance hospital at home. In: Cochrane Database of Systematic Reviews. Wiley 2016. https://doi.org/10.1002/14651858.CD007491.pub2.

16. Leff B, Burton L, Mader SL, Naughton B, Burl J, Inouye SK, et al. Hospital at home: feasibility and outcomes of a program to provide hospital-level care at home for acutely ill older patients. Ann Intern Med. 2005;143:798-808.

17. NHS England. Toolkit for general practice in supporting people with frailty. 2017. https://www.england.nhs.uk/wp-content/uploads/2017/03/toolkitgeneral-practice-frailty.pdf. Accessed 10 Jan 2018.

18. British Geriatric Society. Comprehensive Geriatric Assessment. 2016. http:// www.bgs.org.uk/cga-toolkit/cga-toolkit-category/what-is-cga/cga-what. Accessed 10 Jan 2018.

19. Welsh TJ, Gordon AL, Gladman JR. Comprehensive Geriatric Assessment - a guide for the non-specialist. Int J Clin Pract. 2014;68:290-3.

20. Parker SG, McCue P, Phelps K, McCleod A, Arora S, Nockels K, et al. What is Comprehensive Geriatric Assessment (CGA)? An umbrella review. Age Ageing. 2018:47:149-55.

21. Shepperd S, Cradduck-Bamford A, Butler C, Ellis G, Godfrey M, Gray A, et al. A multi-centre randomised trial to compare the effectiveness of geriatricianled admission avoidance hospital at home versus inpatient admission. Trials. 2017;18:491.

22. Craig P, Dieppe P, Macintyre S, Michie S, Nazareth I, Petticrew M. Developing and evaluating complex interventions: the new Medical Research Council guidance. BMJ. 2008;337:a1655.

23. Moore GF, Audrey S, Barker M, Bond L, Bonell C, Hardeman W, et al. Process evaluation of complex interventions: Medical Research Council guidance. BMJ. 2015; h1258:350.

24. Grant A, Treweek S, Dreischulte T, Foy R, Guthrie B. Process evaluations for cluster-randomised trials of complex interventions: a proposed framework for design and reporting. Trials. 2013;14:15.

25. Hawe P, Shiell A, Riley T. Complex interventions: how 'out of control' can a randomised controlled trial be? BMJ. 2004;328:1561-3.

26. Damschroder L, Aron DC, Keith RE, Kirsh SR, Alexander JA, Lowery JC. Fostering implementation of health services research findings into practice: a consolidated framework for advancing implementation science. Implement Sci. 2009;4. https://doi.org/10.1186/1748-5908-4-50.

27. Kirk MA, Kelley C, Yankey N, Birken SA, Abadie B, Damschroder L. A systematic review of the use of the Consolidated Framework for Implementation Research. Implement Sci. 2016;11. https://doi.org/10.1186/s13012-016-0437-z.

28. Davidoff F, Dixon-Woods M, Leviton L, Michie S. Demystifying theory and its use in improvement. BMJ Qual Saf. 2015. https://doi.org/10.1136/bmjqs2014-003627.

29. Cresswell JW. Qualitative inquiry and research design: choosing among five perspectives. London: Sage; 2007.

30. Patton MQ. Qualitative evaluation and research methods. Newbury Park: SAGE Publications, Inc:; 1990.

31. Palinkas LA, Horwitz SM, Green CA, Wisdom JP, Duan N, Hoagwood K. Purposeful sampling for qualitative data collection and analysis in mixed method implementation research. Admin Pol Ment Health. 2015;42:533-44.

32. Kemper EA, Stringfield S, Teddlie C. Mixed methods sampling strategies in social science research. In: Tashakkori A, Teddlie C, editors. Handbook of mixed methods in the social and behavioral sciences. Thousand Oaks: Sage; 2003. p. 273-96. 
33. Department of Health. Mental Capacity Act Code of Practice. Dep Const Aff Lond TSO. 2005.

34. Scottish Parliament. Adults with Incapacity (Scotland) Act 2000. https:// www.gov.scot/Publications/2008/03/25120154/1. Accessed 9 Oct 2018

35. Age UK. Frailty: Language and Perceptions. 2015. https:/www.nursingtimes. net/Journals/2015/07/23/o/e/e/Age-UK\%2D\%2D-BGS\%2D\%2D-Frailty-FinalReport.pdf. Accessed 16 Jan 2018

36. Church M, Watts S. Assessment of mental capacity: a flow chart guide. Psychiatrist. 2007;31:304-7

37. Berger JT. Is best interests a relevant decision making standard for enrolling non-capacitated subjects into clinical research? J Med Ethics. 2011;37:45-9.

38. Hydén L-C. Narrative collaboration and scaffolding in dementia. J Aging Stud. 2011;25:339-47.

39. Greenwood N. Reflections of a researcher interviewing older people: Nan Greenwood discusses three issues of research-recruitment, terminology and the end of the research relationship-and why researchers should be sensitive to how participants may be affected by the process. Nurs Older People. 2009;21:30-2.

40. Birks Y, Harrison R, Bosanquet K, Hall J, Harden M, Entwistle V, et al. Detailed statement for reflexivity. PubMed Health. 2014. https:/www.ncbi.nlm.nih. gov/pubmedhealth/PMH0081265/. Accessed 25 Mar 2018.

41. Berger R. Now I see it, now I don't: researcher's position and reflexivity in qualitative research. Qual Res. 2015;15:219-34.

42. Hsieh H-F, Shannon SE. Three approaches to Qualitative Content Analysis. Qual Health Res. 2005;15:1277-88.

43. Braun V, Clarke V. Using thematic analysis in psychology. Qual Res Psychol. 2006;:77-101

44. Ritchie J, Lewis J. Qualitative research practice: a guide for social science students and researchers. London: SAGE; 2003.

45. Gale NK, Heath G, Cameron E, Rashid S, Redwood S. Using the framework method for the analysis of qualitative data in multi-disciplinary health research. BMC Med Res Methodol. 2013;13:117.

46. Nowell LS, Norris JM, White DE, Moules NJ. Thematic analysis: striving to meet the trustworthiness criteria. Int J Qual Methods. 2017;16:160940691773384.

Ready to submit your research? Choose BMC and benefit from:

- fast, convenient online submission

- thorough peer review by experienced researchers in your field

- rapid publication on acceptance

- support for research data, including large and complex data types

- gold Open Access which fosters wider collaboration and increased citations

- maximum visibility for your research: over $100 \mathrm{M}$ website views per year

At $\mathrm{BMC}$, research is always in progress.

Learn more biomedcentral.com/submissions 